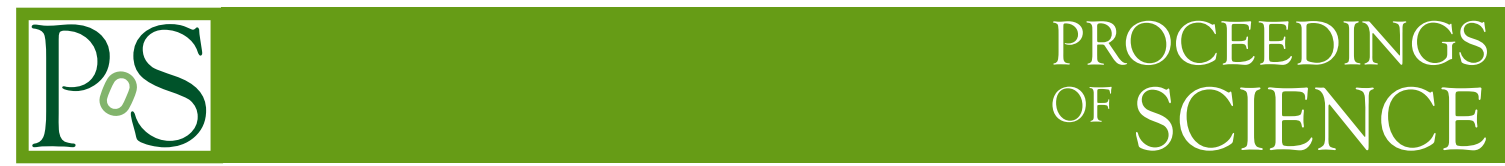

\title{
Powerful relativistic jets in narrow-line Seyfert 1 galaxies (review)
}

\section{Luigi Foschini ${ }^{* \dagger}$}

INAF - Osservatorio Astronomico di Brera, Via E. Bianchi 46, 23807, Merate (LC), Italy

E-mail: luigi.foschini@brera.inaf.it

The recent detection of high-energy gamma rays from Narrow-Line Seyfert 1 Galaxies has confirmed that also this type of active galactic nuclei can generate powerful relativistic jets. I outline the evolution of the knowledge in this research field and the implications on the unification of relativistic jets at all scales.

Nuclei of Seyfert galaxies and QSOs - Central engine \& conditions of star formation November 6-8, 2012

Max-Planck-Insitut für Radioastronomie (MPIfR), Bonn, Germany

\footnotetext{
* Speaker.

${ }^{\dagger}$ I would like to thank the organizers, and particularly S. Komossa, for having realized a pleasant and scientifically fruitful workshop, and for the financial support.
} 


\section{Early times}

In early-mid nineties, the unified model of active galactic nuclei (AGN) reached a reliable shape as the result of the efforts of many researchers (e.g. [50, 6, 5, 81]). Narrow-Line Seyfert 1 Galaxies (NLS1), recognized as a peculiar class of AGN in the second half of eighties ([68, 42], but see also [35]), were initially not included in the unified model. In the Table 1 of Urry \& Padovani [81] NLS1s are not present, but would have been likely to be placed in the radio-quiet branch, under the column Type 0 or between Type 1 and Type 0 . Later works included NLS1s and placed them in a region of radio-quiet sources characterized by low mass of the central black hole, high accretion luminosity, and hosted by spiral galaxies (e.g. [11], Fig. 7).

However, also a population of radio-loud NLS1s (RLNLS1s) emerged slowly, one at a time. The first RLNLS1 - PKS 0558-504 $(z=0.137)$ - was discovered in 1986 by Remillard et al. [76] during the identification of eight sources detected by HEAO 1. It was not yet recognized as NLS1 (the seminal paper by Osterbrock \& Pogge [68] was published just the previous year), but they wrote: "One of the I Zw 1 types, the previously unidentified radio source PKS 0558504 , is a QSO with unusually narrow hydrogen lines for a high-luminosity object $\left(M_{v}=-25.1\right)$." Later, again Remillard et al. [77], reported strong and rapidly variable X-ray emission $(+67 \%$ in 3 minutes) from the same source detected by Ginga. The measured energies and time scales required relativistic beaming to be reasonably explained. A second candidate - RGB J0044+193 $(z=0.181)$ - was found in 1999 while searching for X-ray selected BL Lac Objects in the ROSAT-Green Bank survey (RGB, [78]), but it was later suggested that the radio detection could be spurious, thus restoring the radio-quiet classification [57]. More RLNLS1s were discovered in these years. Grupe et al. [44] in 2000 reported about RX J0134.2-4258 $(z=0.237)$ : ROSAT observations indicated spectral variability (fainter when softer), with a variable hard component, confirmed also by ASCA. They proposed three possible explanations: warm absorber, corona, or relativistic jet. The third case followed soon: PKS 2004-447 $(z=0.24)$ was discovered by Oshlack et al. [67] (see also [34]). About one dozen of RLNLS1s were found in a survey done by Zhou \& Wang [84].

The advent of the Sloan Digital Sky Survey (SDSS) in early 2000s determined a major change in this research field. The public availability of thousands of optical spectra allowed the crosscorrelation with radio catalogues to search for more candidates. Particularly, it is worth mentioning H. Y. Zhou, who gave many important contributions by discovering, with his colleagues, several RLNLS1s [85-87, 83]. Specifically, they reported about the radio properties of three sources SDSS J094857.3+002225 $(z=0.585)$ [85], SDSS J084957.97+510829.0 $(z=0.584)$ [86], and $1 \mathrm{H} 0323+342(z=0.061)$ [87] - that were very similar to blazars (flat or inverted radio spectrum, high brightness temperature), suggesting the presence of a relativistic jet viewed at small angles. Interestingly, all these three sources were later detected at high-energy $\gamma$ rays [4]. These works triggered deeper studies at radio frequencies of some RLNLS1s $[16,17]$, where the typical radio properties of relativistic jets were confirmed, and, in addition, the radio morphology resulted to be very compact on parsec scale.

A first attempt to search for emission at TeV energies from RLNLS1s was done by Falcone et al. [22] in 2004 by using the ground-based Whipple telescope. However, the aim of that study was to detect at $\gamma$ rays some candidates of the elusive population of high-frequency peaked flat-spectrum radio quasars (HFSRQs) postulated by Padovani et al. [69, 70] to challenge the so-called "blazar 
sequence" $[33,36]$. According to the latter, blazars follow a sequence linking the frequency of the synchrotron peak to the jet power: the greater the power, the lower the synchrotron peak frequency, and vice versa. Therefore, to find a high-power blazar with a high synchrotron peak frequency (in the UV/X-rays) would have been a major break in the sequence. Among the candidates in the list of Falcone there were two RLNLS1s - 1H 0323+342 and SDSS J162901.30+400759.9 - but not recognized as such. It was likely a misinterpretation of the strong soft X-ray emission of NLS1s, due to the accretion disk, which instead was considered as an indication of a synchrotron peak at $\mathrm{X}$-rays ${ }^{1}$. Anyway, the search of $\mathrm{TeV}$ emission was negative, but intriguing. In the words by Falcone et al. [22]: "No significant emission has been detected from any of the candidate sources in this initial survey. There was marginal evidence of a rate increase observed in the B2 $0321+33$ [alias $1 \mathrm{H} 0323+342]$ light curve, but the statistical significance of this increase is $2.5 \sigma$ (post-trial significance), which could be accounted for by a statistical fluctuation".

In mid-2000s, two more surveys were done by Whalen et al. [82] and Komossa et al. [52], who did also a specific paper on one source of her list [53]. The former survey was done by using the FIRST Bright Quasar Survey (FBQS) and the authors conclude that "except for their radio properties, radio-selected NLS1 galaxies do not exhibit significant differences from traditional NLS1 galaxies. Our results are also in agreement with previous studies suggesting that NLS1 galaxies have small black hole masses that are accreting very close to the Eddington rate" [82]. Instead, Komossa et al. studied a more heterogeneous and small sample and concluded that "while properties of most sources (with two to three exceptions) generally do not favor relativistic beaming, the combination of accretion mode and spin may explain the observations" [52]. In 2008, there was the SDSS sample of 23 RLNLS1s by Yuan et al. [83]: "Intrinsically, some of them have relatively low radio power and would have been classified as radio-intermediate AGNs. The black hole masses are estimated to be within $10^{6}-10^{8} M_{\odot}$, and the Eddington ratios close to unity, as in normal NLS1 AGNs. The results imply that radio-loud AGNs may be powered by black holes of moderate masses $\left(\sim 10^{6}-10^{7} M_{\odot}\right)$ accreting at high rates".

On the basis of the radio morphology and spectra, Komossa et al. [52] also suggested that some RLNLS1s of their sample could be similar to Compact Steep Spectrum (CSS) radio sources. Gallo et al. [34] found that one clear example of RLNLS1/CSS was PKS 2004-447. Although Komossa et al. [52] cited this source, they did not include in their sample on the basis of a weak FeII bump, which in turn would suggest a classification of this AGN as narrow-line radio galaxy or type II AGN. However, Gallo et al. [34] noted that there is no formal threshold for the intensity of Fe II, thus holding the classification as RLNLS1.

Meanwhile, another important player introduced itself in this game: the Swift satellite, launched on November 2004. Again, the policy of use played an important role. The possibility for almost everyone to have even a little snapshot and the immediate public availability of data, made it possible to perform many researches outside the mainstream, as it was the case of RLNLS1s. In the second half of 2000s, the Swift public archive was sufficiently rich of observations on RLNLS1 to perform an early little survey [29]. Some interesting results were found, specifically about 1H $0323+342$ (Fig. 1, left panel): the source had a strong optical-to-X-ray emission from the

\footnotetext{
${ }^{1}$ The HFSRQs were at last found in 2012 by Padovani et al. [71]. See, however, a different interpretation by Ghisellini et al. [37] and Foschini [28].
} 

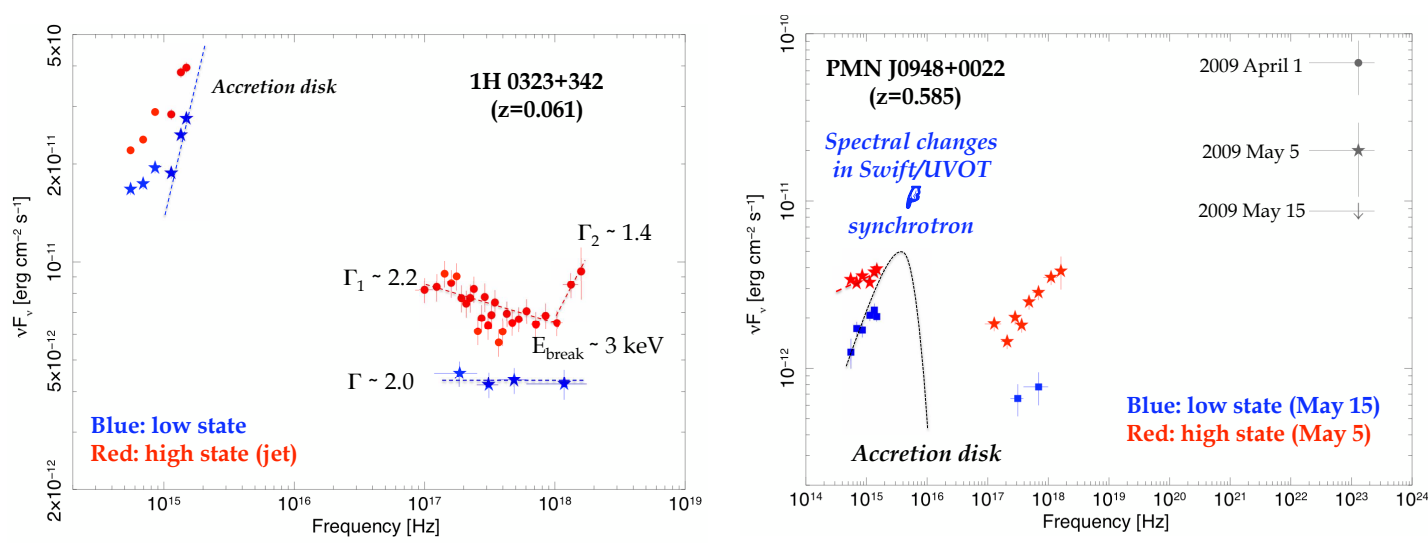

Figure 1: (left panel) Swift (XRT and UVOT) observations of the RLNLS1 1H 0323+342 in two different states. When the jet is not active (blue points, ObsID 00036533007, 21 Dec 2007), the source displays low optical-to-X-ray flux, with a rather flat X-ray photon index. As the jet increases its activity (red points, ObsID 00035372001, 6 Jul 2006), there is a greater optical-to-X-ray flux and the emergence of a hard tail $(\Gamma \sim 1.4)$. See also [29]. (right panel) Swift (XRT and UVOT) and Fermi/LAT observations of the RLNLS1 PMN J0948+0022 in two different states during the 2009 MW campaign [3]. The source was active during 2009 April, with a peak on the day 1. Then, in early May, the activity dropped at all the wavelengths in a coordinated way. See [3] and the text for more details.

accretion disk, but sometimes the jet emerged, resulting in the appearance of a hard tail. This behaviour was confirmed also by the spectral variability at hard X-rays. The source was observed by INTEGRAL in 2004 with a low flux and soft spectrum $\left(F_{20-40 \mathrm{keV}}=2.5 \pm 0.5 \mathrm{mCrab}\right.$; $F_{40-100 \mathrm{keV}}<2.6 \mathrm{mCrab}$ ), while the integration of the Swift/BAT on axis data between 20062008 (exposure $\sim 53 \mathrm{ks})$ resulted in a high flux and hard spectrum $\left(F_{20-40 \mathrm{keV}}<20 \mathrm{mCrab}\right.$; $\left.F_{40-100 \mathrm{keV}}=16 \pm 2 \mathrm{mCrab}\right)$ [29].

Also the hard X-ray detection was affected by a misinterpretation. Indeed, $1 \mathrm{H} 0323+342$ was already present in a few papers on hard X-ray sources published in 2007, before of [29]: two independent catalogs of the IBIS imager onboard the INTEGRAL satellite [7, 54] and one optical-to-X-ray follow-up with Swift of a sample of 34 hard X-ray AGN [58]. However, in all these papers, $1 \mathrm{H} 0323+342$ was not recognized as RLNLS1, but classified as a normal Seyfert 1. In a subsequent paper reporting the "first high-energy observations of narrow-line Seyfert 1s" where "high-energy" here means in the range 17-100 keV - Malizia et al. [59] studied two-three candidate radio-quiet NLS1s discovered by INTEGRAL and Swift/BAT, and with no reference to any rethinking about $1 \mathrm{H} 0323+342$. The latter was included in the INTEGRAL sample only in 2011 by Panessa et al. [73].

\section{The Fermi breakthrough}

A real change in the perception of RLNLS1s came in 2008 with the launch of the Fermi Gamma-ray Space Telescope (hereafter Fermi), but also in this case the emerging of RLNLS1s as a new class of $\gamma$-ray emitting AGN was not straightforward. Indeed, the first RLNLS1 to be detected at MeV-GeV energies - SDSS J094857.3+002225 (alias PMN J0948+0022) [2] - was 
also present among the list of bright sources detected after the first three months of Fermi activity [1]. However, it was still identified as a FSRQ, although the text refers to another specific paper in preparation: "The source PMN J0948+0022, associated with OFGL J0948.3+0019, has a flat radio spectrum but shows an optical spectrum with only narrow emission lines, leading to an 'uncertain' type classification in Roma-BZCat. A detailed analysis of this source is presented in Abdo et al. (2009a)" [the latter being the reference [2] in the present work]. It was indeed in [2] that it was reported the first high-energy $\gamma$-ray detection of a RLNLS1 in an explicit and "conscious" way. The discovery was soon followed by a complementary paper containing additional information to improve the identification [30].

Obviously, these early works linked the $\gamma$-ray source with the RLNLS1 on a statistical basis. Therefore, a multiwavelength (MW) campaign was activated to study in detail the electromagnetic emission of PMN J0948+0022 from radio to $\gamma$ rays [3]. We had sufficient luck to catch coordinated variability at different frequencies. PMN J0948+0022 displayed a moderate $\gamma$-ray activity on 2009 April, with fluxes of the order of a few $\times 10^{-7} \mathrm{ph} \mathrm{cm}^{-2} \mathrm{~s}^{-1}(0.1<E<100 \mathrm{GeV})$. In early May there was a drop of the $\gamma$-ray flux followed by a decrease of the optical-to-X-ray flux as measured by Swift, together with a spectral change in the optical/UV spectrum (Fig. 1, right panel). This was interpreted as a decrease of the jet emission (synchrotron), which left the optical/UV wave band dominated by the thermal emission from the accretion disk. This point of view was confirmed by the fact that a few weeks later, there was the peak of the radio emission as recorded from OVRO, Metsähovi, and Effelsberg ground-based radio telescopes [3], as expected from the classical theory of relativistic jets [10]. Indeed, the blob of plasma has to be compact to be optically thin to $\gamma$ rays, but in this case the lower part of the electromagnetic spectrum (radio frequencies) is self-absorbed. As the blob moves outward, it expands itself, thus becoming optically thick to $\gamma$ rays, but thin to radio emission. Moreover, additional support to this interpretation came from the detection of optical $(V)$ polarization at $\sim 19 \%$ from the Kanata telescope between the end of March and the beginning of April 2009, when the source was moderately active at $\gamma$ rays [47]. All these information concurred to establish a well-grounded association of the Fermi detected $\gamma$-ray source with the RLNLS1 PMN J0948+0022. Other MW campaigns strengthened the association and the similarities of the jet with those of blazars [31,32]. Other optical-infrared observations confirmed violent intranight variability, as expected from AGN with relativistic jets viewed at small angles $[56,49,72]$.

After one year of Fermi operations, the number of $\gamma$-ray detected RLNLS1s increased to four [30], with the addition of $1 \mathrm{H} 0323+342$ (cf [87, 29, 73]), PKS 2004-447 (cf [67, 34, 29]), and PKS 1502+036 (cf [83]). RLNLS1s were then explicitly indicated "as a new class of gammaray active galactic nuclei”, in addition to blazars and radio galaxies [30]. One more RLNLS1, SBS $0846+513$ (cf. [86]) was later detected because of an outburst ([23, 20, 14]), indicating that the probability of detection at $\gamma$ rays is still biased by the sensitivity of the instrument. The above cited sources have all been detected at $\gamma$ rays with high significance (TS $>25$, see [62] for the definition of TS, or $\gtrsim 5 \sigma$ ). However, it is worth noting that there are also several detections at low significance $(9<T S<25$ or $3<\sigma<5$ ), so that there is presently one dozen of RLNLS1s detected at $\mathrm{MeV}-\mathrm{GeV}$ energies [23] $\left(^{2}\right)$. The number is expected to increase, depending on the activity of

\footnotetext{
${ }^{2}$ See the updated list at the web page http://tinyurl.com/gnls1s
} 
the sources. The ability of Fermi to scan all the sky every three hours guarantees a continuous monitoring.

\section{The parent population}

One dozen of RLNLS1s with the jet viewed at small angles (five, in the most conservative hypothesis) implies $\sim 2 \Gamma^{2}$ times parent sources, i.e. with the jet viewed at large angles. $\Gamma$, the bulk Lorentz factor of the jet, is generally $\sim 10$, which in turn means at least $\sim 10^{3}$ parent sources. The problems is that it is quite difficult to find them.

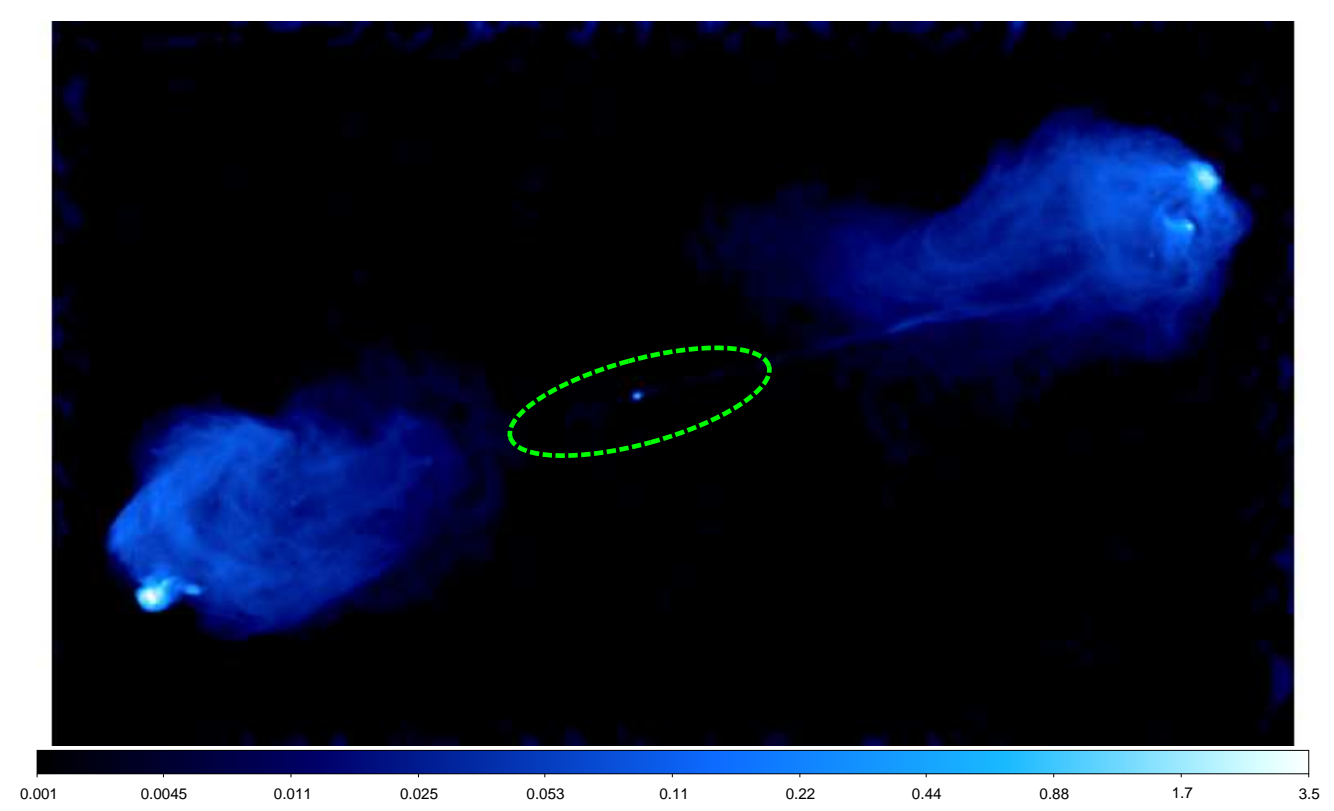

Figure 2: Cygnus A as observed by the Very Large Array (VLA) at $6 \mathrm{~cm}$ [74]. Personal elaboration of the original FITS file. See text for details.

Basically, there are three hypotheses:

1. The first one - rather obvious - is to search simply for RLNLS1s with jet viewed at large angles. This could be not so easy, because early studies indicated a very compact structure for beamed NLS1s [16, 17, 45, 40]. Nevertheless, some interesting cases are emerging, like PKS 0558-504, where Gliozzi et al. [41] found a bipolar radio jet $46 \mathrm{kpc}-$ long (projected) likely viewed at $30^{\circ}-45^{\circ}$. Three more candidates have been found by Doi et al. [18].

2. The second hypothesis is based on the fact that RLNLS1s are extremely compact at radio frequencies. If there is no extended emission, then it is possible that these sources are radio quiet when observed at large angles. To better understand this hypothesis, have a look at one well-known radio image of the radio galaxy Cygnus A (Fig. 2). Most of the radio emission comes from the hot spots and the core, while the jet is almost invisible, an indication of its high efficiency. Now think to a similar source, but without the hot spots. NLS1s are thought 
to be young AGN - cf $[43,60,61,52]$ - and therefore one could expect that the spots have not yet developed. Roughly, the source is now limited to what is inside the dashed ellipse in Fig. 2. The source observed pole-on has a compact morphology with a high brightness temperature core. When it is observed edge-on, the radio emission - no more enhanced by the special relativity effects - becomes much fainter and such a source might seem radio quiet. In this case, the parent population could be that of the radio-quiet NLS1s. Radio quiet, but not radio silent, as proved by [80]. Indeed, some jet-like radio structures have been found also in radio-quiet NLS1s [39, 66, 19].

3. The third and last option is based on the hypothesis that the broad-line region has a disk-like shape [15]. This means that when the source is observed pole-on, there is no component of motion directed toward the observer and, hence, no Doppler broadening. When observed edge-on, the Doppler broadening is present and therefore the line profile is broad. Therefore, in this case, it is necessary to search among the usual radio galaxies, but hosted by disk/spiral galaxies, as NLS1s generally have that type of host ${ }^{3}$. Some examples have been found, e.g. $[12,21,63,75,51,66,65]$. It is worth noting that a systematic study on the morphology of the host galaxy of a flux-limited sample of radio galaxies ( $2 \mathrm{Jy}$ ) resulted in the evidence that $12 \%$ of the sources are hosted by disk galaxies [48].

The parent population presently remains an open question. It is not yet clear if one of the above three will win or if it will result a mixture including a bit of all the three or, even, in a yet-to-be-made fourth hypothesis.

\section{The role of RLNLS1s in the unification of jets at all scales}

The discovery of powerful relativistic jets in RLNLS1s is not "simply" the addition of one more class of $\gamma$-ray AGN. It has deep implications in the unification of jets at all scales. RLNLS1s are different from blazars in many aspects, but the jets seem to be almost the same, as proved by the MW campaigns [3, 31, 32]. Indeed, some researchers (e.g. [14]) suggested that RLNLS1s might be blazars in a early stage of their life. While there is agreement on the physical properties of the jets in blazars and RLNLS1s, to name all these sources simply as blazars could be strongly misleading. The reason supporting the inclusion of RLNLS1 in the blazar name is that some researchers refer the term simply to the jet emission boosted by the special relativity (somehow derived from the verb "to blaze"), disregarding any information on the AGN, environment, and host. Ulrich et al. [79] wrote: "In radio-loud AGN seen at small angles to the axis of the jet, the highly nonthermal radiation produced in the jet is strongly amplified by relativistic beaming and dominates the observed continuum. In these sources, called blazars, variability is the most violent and affects the whole electromagnetic range from the radio to the gamma-ray band.". Burbidge \& Hewitt [13] wrote that "at the dinner at the end of that meeting [the well-known Pittsburgh conference on BL Lac Objects held in 1978], Spiegel coined the term 'blazar' a pictorial term which he proposed be applied to rapidly variable objects some of which, but not all, can also be classified as BL Lac objects." The problem is not the term itself: since it is now evident that the

\footnotetext{
${ }^{3}$ Blazars and radio galaxies are instead hosted by elliptical galaxies.
} 
powerful jets in RLNLS1s are the same of blazars, it might be useful to speak about all these sources dominated by the jet emission as blazars, given the above cited definitions $\left({ }^{4}\right)$.

The problem is that historically blazars refer to BL Lac Objects and FSRQs. According to Wikipedia, the Spiegel's term was the result of the contraction of the words BL Lac objects and Optically Violent Variable (OVV) quasars $\left({ }^{5}\right)$. However, BL Lac Objects and FSRQs are AGN different from RLNLS1s in several ways. Therefore, the simple inclusion of RLNLS1s in the blazar semantic field results in missing some important new information specifically linked to these peculiar sources, which can be particularly useful for the unification of jets at all scales. For example, one is the confirmation of what already told Roger Blandford at the Pittburgh 1978 conference on BL Lac Objects: "As the continuum [jet] emission is proposed to originate in the central $10 \mathrm{pc}, \mathrm{I}$ don't think the nature of the surrounding object is particularly relevant to the model" [9]. The fact that powerful relativistic jets develop also in the environment of RLNLS1s, which is different from that of blazars, is one more arrow for the Blandford's bow. Another important information is the break down of the mass requirement of the central accreting object to develop a jet in AGN (e.g. [55]): since the masses of the central black hole of RLNLS1s are smaller than those of blazars, this requirement is no more valid, making thus possible to perform the unification (such mass requirement was not present on Galactic scale).

Another change in the common knowledge is required to unify efficiently the jets at all scales and refers to the analogy with compact objects on Galactic scale. Traditionally, NLS1s have been considered as the large scale version of stellar mass black holes in soft/high state, because of the high-accretion rate (e.g. [41]). However, what it matters is the mass not the accretion: when displaying the jet power as a function either of the mass of the central compact object or the disk luminosity, two branches resulted (see Fig. 3 in [24]). One dependent on the mass (RPD, radiation-pressure dominated regime) and the other dependent on the accretion (GPD, gas-pressure dominated regime), which in turn are in agreement with the expectations from the theory of jets $[8,64,38]$. RLNLS1s are placed in the RPD branch. Another way to see this effect is displayed in Fig. 2 of [26]: it is evident that the RLNLS1s (low-mass AGN) make a branch similar to that of neutron stars on Galactic scale (low-mass binaries). It is also worth stressing that without powerful jets in RLNLS1s, the low-mass AGN branch would be missing, thus making impossible a unification of jets at all scales, despite the interesting attempts made in the past. Instead, now with RLNLS1, one can rescale the jet power according to the mass of the compact object ( $M^{1.4}$ according to [46]), making it possible to merge the Galactic and extragalactic jets [25, 26]. It remains to understand a slight dependence on the disk luminosity (cf. [25]), which in turn depends on our understanding of the disk structure and its efficiency in the conversion of the gravitational potential energy into radiation.

Another open question is the role of the spin of the compact object, although rather than the spin alone, it is necessary to study also how to measure the angular speed of the magnetic field lines (cf. [8]). As it will be possible to measure both angular velocities, perhaps it will be possible to understand the division between AGN with or without relativistic jets (e.g. [27]).

\footnotetext{
${ }^{4}$ I myself coauthored in 2009 a paper titled: “Blazar nuclei in radio-loud narrow-line Seyfert 1?” [29].

5 http://en.wikipedia.org/wiki/Blazar
} 


\section{Conclusions}

The class of RLNLS1 is an important piece in our understanding of relativistic jets at all scales. Although each work has given an important contribution to the present mosaic, the real breakthrough occurred in 2008 with the detection of high-energy $\gamma$ rays with Fermi/LAT and the subsequent MW campaigns. This definitely proved the presence of powerful relativistic jets in this type of AGN and allowed to set an important step toward the unification of relativistic jets.

\section{References}

[1] A. A. Abdo et al. (LAT Collaboration): Bright active galactic nuclei source list from the first three months of the Fermi Large Area Telescope all-sky survey, ApJ 700 (2009), 597.

[2] A. A. Abdo et al. (LAT Collaboration): Fermi/Large Area Telescope discovery of gamma-ray emission from a relativistic jet in the narrow-line quasar PMN J0948+0022, ApJ 699 (2009) 976.

[3] A. A. Abdo et al. (LAT Collaboration): Multiwavelength monitoring of the enigmatic narrow-line Seyfert 1 PMN J0948+0022 in 2009 March-July, ApJ 707 (2009) 727.

[4] A. A. Abdo et al. (LAT Collaboration): Radio-loud narrow-line Seyfert 1 as a new class of gamma-ray active galactic nuclei, ApJ 707 (2009) L142.

[5] R. Antonucci: Unified models for active galactic nuclei and quasars, ARA\&A 31 (1993) 473.

[6] P. D. Barthel: Is every quasar beamed?, ApJ 336 (1989) 606.

[7] A. J. Bird et al.: The Third IBIS/ISGRI Soft Gamma-Ray Survey Catalog, ApJS 170 (2007) 175.

[8] R. D. Blandford \& R. L. Znajek: Electromagnetic extraction of energy from Kerr black holes, MNRAS 179 (1977) 433.

[9] R. D. Blandford \& M. J. Rees: Some comments on radiation mechanisms in Lacertids. In: Proceedings of the Pittsburgh Conference on BL Lac Objects, University of Pittsburgh (1978) p. 328.

[10] R. D. Blandford \& A. Königl: Relativistic jets as compact radio sources, ApJ 232 (1979) 34.

[11] T. A. Boroson: Black hole mass and Eddington ratio as drivers for the observable properties of radio-loud and radio-quiet QSOs, ApJ 565 (2002) 78.

[12] A. Brunthaler et al.: III Zw 2, the first superluminal jet in a Seyfert galaxy, A\&A 357 (2000) L45.

[13] G. Burbidge \& A. Hewitt: BL Lacertae Objects and Rapidly Variable QSOs - An Overview, in: Variability of Blazars, Proceedings from the Conference in honour of the 100th anniversary of the birth of Yrjö Väisälä., eds E. Valtaoja \& M. Valtonen, Cambridge University Press, Cambridge (1992), p. 4.

[14] F. D'Ammando et al.: SBS 0846+513: a new $\gamma$-ray-emitting narrow-line Seyfert 1 galaxy, MNRAS 426 (2012) 317.

[15] R. Decarli et al.: Are the black hole masses in narrow-line Seyfert 1 galaxies actually small?, MNRAS 386 (2008) L15.

[16] A. Doi et al.: VLBI Observations of the most radio-loud narrow-line quasar SDSS J094857.3+002225, PASJ 58 (2006) 829.

[17] A. Doi et al.: Japanese VLBI network observations of radio-loud narrow-line Seyfert 1 galaxies, PASJ 59 (2007) 703. 
[18] A. Doi et al.: Kiloparsec-scale Radio Structures in Narrow-line Seyfert 1 Galaxies, ApJ 760 (2012) 41.

[19] A. Doi et al.: Very Long Baseline Array Imaging of Parsec-scale Radio Emissions in Nearby Radio-quiet Narrow-line Seyfert 1 Galaxies, ApJ, (2013), accepted for publication (arXiv:1301.4758).

[20] D. Donato \& J. S. Perkins (LAT Collaboration): Fermi LAT detection of a GeV flare from the Radio-Loud Narrow-Line Sy1 SBS 0846+513, The Astronomer's Telegram, (2011), \# 3452.

[21] B. H. C. Emonts et al.: Enormous disc of cool gas surrounding the nearby powerful radio galaxy NGC612 (PKSO131-36), MNRAS 387 (2008) 197.

[22] A. D. Falcone et al.: A search for TeV gamma-ray emission from high-peaked flat-spectrum radio quasars using the Whipple Air Cerenkov telescope, ApJ 613 (2004) 710.

[23] L. Foschini: Evidence of powerful relativistic jets in narrow-line Seyfert 1 galaxies, in: Narrow-Line Seyfert 1 Galaxies and Their Place in the Universe, eds. L. Foschini, M. Colpi, L. Gallo, D. Grupe, S. Komossa, K. Leighly, \& S. Mathur, (2011), PoS (NLS1) 024

[24] L. Foschini: Accretion and jet power in active galactic nuclei, Res. Astron. Astrophys. 11 (2011) 1266.

[25] L. Foschini: Powerful relativistic jets in spiral galaxies, Int. J. Mod. Phys. Conf. Series 8 (2012) 172.

[26] L. Foschini: $\gamma$-ray emission from Narrow-Line Seyfert 1 galaxies and implications on the jets unification, in: High-Energy Gamma-Ray Astronomy: 5th International Meeting on High Energy Gamma-Ray Astronomy, eds F. A. Aharonian, W. Hofmann, \& F. M. Rieger, AIP Conf. Proc. 1505 (2012) 574.

[27] L. Foschini: The Gravitational Asynchronous Machine, (2012), arXiv: 1205.3128.

[28] L. Foschini: Report from the Bologna High-Energy Meeting (BOHEME), Yet Another Blog, May 28, 2012 (http: / / tinyurl. com/b7lkxwg).

[29] L. Foschini et al.: Blazar nuclei in radio-loud narrow-line Seyfert 1?, Adv. Space Res. 43 (2009) 889.

[30] L. Foschini et al. (LAT Collaboration): Fermi/LAT discovery of gamma-ray emission from a relativistic jet in the narrow-line Seyfert 1 quasar PMN J0948+0022, in: Accretion and Ejection in AGNs: A Global View, eds L. Maraschi, G. Ghisellini, R. Della Ceca, \& F. Tavecchio, ASP Conference Series 427 (2010) 243.

[31] L. Foschini et al.: The first gamma-ray outburst of a narrow-line Seyfert 1 galaxy: the case of PMN J0948+0022 in 2010 July, MNRAS 413 (2011) 1671.

[32] L. Foschini et al.: Radio-to- $\gamma$-ray monitoring of the narrow-line Seyfert 1 galaxy PMN J0948+0022 from 2008 to 2011, A\&A $\mathbf{5 4 8}$ (2012) A106.

[33] G. Fossati et al.: A unifying view of the spectral energy distributions of blazars, MNRAS 299 (1998) 433.

[34] L. Gallo et al.: The spectral energy distribution of PKS 2004-447: a compact steep-spectrum source and possible radio-loud narrow-line Seyfert 1 galaxy, MNRAS 370 (2006) 245.

[35] C. M. Gaskell: Reddening of the Narrow-Line regions of Active Galactic Nuclei and the Intrinsic Balmer decrement II, Astroph. Lett. 24 (1984) 43.

[36] G. Ghisellini et al.: A theoretical unifying scheme for gamma-ray bright blazars,MNRAS 301 (1998) 451. 
[37] G. Ghisellini et al.: Blue Fermi flat spectrum radio quasars, MNRAS 425 (2012) 1371.

[38] P. Ghosh \& M. A. Abramowicz: Electromagnetic extraction of rotational energy from disc-fed black holes: the strength of the Blandford-Znajek process, MNRAS 292 (1997) 887.

[39] M. Giroletti \& F. Panessa: The Faintest Seyfert Radio Cores Revealed by VLBI, ApJ 706 (2009) L260.

[40] M. Giroletti et al.: Global e-VLBI observations of the gamma-ray narrow line Seyfert 1 PMN J0948+0022, A\&A $\mathbf{5 2 8}$ (2011) L11.

[41] M. Gliozzi et al.: A Panchromatic View of PKS 0558-504: An Ideal Laboratory to Study the Disk-Jet Link, ApJ 717 (2010) 1243.

[42] R. W. Goodrich: Spectropolarimetry of “Narrow-Line” Seyfert 1 Galaxies, ApJ 342 (1989) 224.

[43] D. Grupe et al.: New bright soft X-ray selected ROSAT AGN. II. Optical emission line properties, A\&A 350 (1999) 805.

[44] D. Grupe et al.: The enigmatic soft X-ray AGN RX J0134.2-4258, A\&A 356 (2000) 11.

[45] M. Gu \& Y. Chen: The Compact Radio Structure of Radio-loud Narrow Line Seyfert 1 Galaxies, AJ 139 (2010) 2612.

[46] S. Heinz \& R. A. Sunyaev: The non-linear dependence of flux on black hole mass and accretion rate in core-dominated jets, MNRAS 343 (2003) L59.

[47] Y. Ikejiri et al.: Photopolarimetric Monitoring of Blazars in the Optical and Near-Infrared Bands with the Kanata Telescope. I. Correlations between Flux, Color, and Polarization, PASJ 63 (2011) 639.

[48] K. Inskip et al.: A near-IR study of the host galaxies of 2 Jy radio sources at $0.03<z<0.5-I$. The data, MNRAS 407 (2010) 1739.

[49] N. Jiang et al.: Rapid Infrared Variability of Three Radio-loud Narrow-line Seyfert 1 Galaxies: A View from the Wide-field Infrared Survey Explorer, ApJ 759 (2012) L31.

[50] W. C. Keel: Inclination effects on the recognition of Seyfert galaxies, AJ 85 (1980) 198.

[51] W. C. Keel et al.: The Spiral Host Galaxy of the Double Radio Source 0313-192, AJ 132 (2006) 2233.

[52] S. Komossa et al.: Radio-loud narrow-line type 1 quasars, AJ 132 (2006) 531.

[53] S. Komossa et al.: The radio-loud narrow-line quasar SDSS J172206.03+565451.6, ApJ 639 (2006) 710 .

[54] R. Krivonos et al.: INTEGRAL/IBIS all-sky survey in hard X-rays, A\&A 475 (2007) 775.

[55] A. Laor: On black hole masses and radio loudness in active galactic nuclei, ApJ 543 (2000) L111.

[56] H. Liu et al.: Violent Intranight Optical Variability of a Radio-loud Narrow-line Seyfert 1 Galaxy: SDSS J094857.3+002225, ApJ 715 (2010) L113.

[57] T. J. Maccarone et al.: Was the narrow line Seyfert 1 RGB J0044+193 ever radio loud?, A\&A 433 (2005) 531 .

[58] A. Malizia et al.: Swift XRT Observation of 34 New INTEGRAL IBIS AGNs: Discovery of Compton-Thick and Other Peculiar Sources, ApJ 668 (2007) 81.

[59] A. Malizia et al.: First high-energy observations of narrow-line Seyfert 1s with INTEGRAL/IBIS, MNRAS 389 (2008) 1360.

[60] S. Mathur: Narrow-line Seyfert 1 galaxies and the evolution of galaxies and active galaxies, MNRAS 314 (2000) L17. 
[61] S. Mathur et al.: Supermassive black holes, pseudobulges, and the Narrow-Line Seyfert 1 Galaxies, ApJ 754 (2012) 146.

[62] J. R. Mattox et al.: The Likelihood Analysis of EGRET Data, ApJ 461 (1996) 396.

[63] I. M. McHardy et al.: Hubble Space Telescope Observations of the BL Lac Object PKS:1413+135: The Host Galaxy Revealed, MNRAS 268 (1994) 681.

[64] R. Moderski \& M. Sikora: On black hole evolution in active galactic nuclei, MNRAS 283 (1996) 854.

[65] R. Morganti et al.: PKS 1814-637: a powerful radio-loud AGN in a disk galaxy, A\&A 535 (2011) A97.

[66] C. G. Mundell et al.: Radio Variability in Seyfert Nuclei, ApJ 703 (2009) 802.

[67] A. Y. K. N. Oshlack, R. L. Webster \& M. T. Whiting: A very radio loud narrow-line Seyfert 1: PKS 2004-447, ApJ 558 (2001) 578.

[68] D. E. Osterbrock \& R. W. Pogge: The spectra of Narrow-Line Seyfert 1 Galaxies, ApJ 297 (1985) 166.

[69] P. Padovani et al.: BeppoSAX observations of synchrotron X-ray emission from radio quasars, ApJ 581 (2002) 895.

[70] P. Padovani et al.: What types of jets does Nature make? A new population of radio quasars, ApJ 588 (2003) 128.

[71] P. Padovani, P. Giommi \& A. Rau: The discovery of high-power high synchrotron peak blazars, MNRAS 422 (2012) L48.

[72] V. S. Paliya et al.: Intranight optical variability of $\gamma$-ray-loud narrow-line Seyfert 1 galaxies, MNRAS 428 (2013) 2450.

[73] F. Panessa et al.: Narrow-line Seyfert 1 galaxies at hard X-rays, MNRAS 417 (2011) 2426.

[74] R. A. Perley et al.: The jet and filaments in Cygnus A, ApJ 285 (1984) L35.

[75] E. S. Perlman et al.: The Apparent Host Galaxy of PKS 1413+135: Hubble Space Telescope, ASCA, and Very Long Baseline Array Observations, AJ 124 (2002) 2401.

[76] R. A. Remillard et al.: The discovery of eight bright active galactic nuclei observed with the HEAO 1 scanning modulation collimator, ApJ 301 (1986) 742.

[77] R. A. Remillard et al.: A rapid energetic X-ray flare in the quasar PKS 0558-504, Nature 350 (1991) 589.

[78] J. Siebert et al.: An ASCA observation of the radio-loud narrow-line Seyfert 1 galaxy RGB J0044+193, A\&A 348 (1999) 678.

[79] M.-H. Ulrich, L. Maraschi, \& C. M. Urry: Variability of active galactic nuclei, Ann. Rev. Astron. Astrophys. 35 (1997) 445.

[80] J. S. Ulvestad et al.: Radio properties of Narrow-Line Seyfert 1 Galaxies, AJ 109 (1995) 81.

[81] C. M. Urry \& P. Padovani: Unified schemes for radio-loud active galactic nuclei, PASP 107 (1995) 803.

[82] D. J. Whalen et al.: Optical properties of radio-selected narrow-line Seyfert 1 galaxies, AJ 131 (2006) 1948.

[83] W. Yuan et al.: A population of radio-loud narrow-line Seyfert 1 galaxies with blazar-like properties?, ApJ 685 (2008) 801. 
[84] H. Y. Zhou \& T. G. Wang: Properties of broad band continuum of Narrow Line Seyfert 1 Galaxies, Ch. J. Astron. Astrophys. 2 (2002) 501.

[85] H. Y. Zhou et al.: SDSS J094857.3+002225: A very radio loud, narrow-line quasar with relativistic jets?, ApJ 584 (2003) 147.

[86] H. Y. Zhou et al.: The hybrid nature of 0846+51W1: a BL Lac Object with a Narrow Line Seyfert 1 Nucleus, Ch. J. Astron. Astrophys. 5 (2005) 41.

[87] H. Y. Zhou et al.: A narrow-line Seyfert 1-blazar composite nucleus in 2MASX J0324+3410, ApJ 658 (2007) L13. 